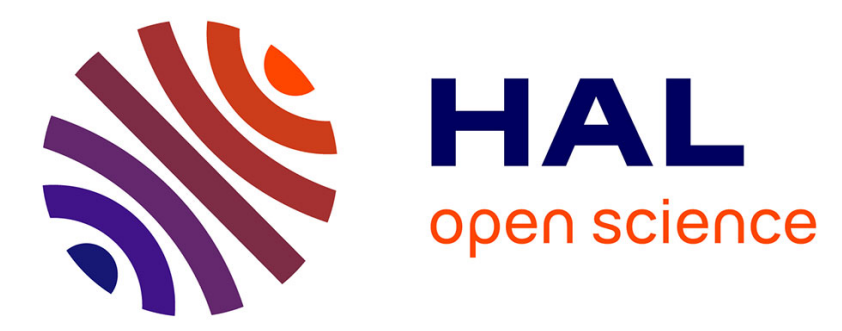

\title{
A Low-Cost System for Indoor Motion Tracking of Unmanned Aerial Vehicles
}

Sylvain Bertrand, Julien Marzat, Mathieu Carton, Cyril Chaix, Paul Varela, Renan Waroux, Guillaume de Ferron, Rosye Laurel

\section{- To cite this version:}

Sylvain Bertrand, Julien Marzat, Mathieu Carton, Cyril Chaix, Paul Varela, et al.. A Low-Cost System for Indoor Motion Tracking of Unmanned Aerial Vehicles. Seventh IEEE International Conference on Intelligent Sensors, Sensor Networks and Information Processing, ISSNIP 2011, Dec 2011, Adelaide, Australia. hal-00659152

\section{HAL Id: hal-00659152 https://hal.science/hal-00659152}

Submitted on 12 Jan 2012

HAL is a multi-disciplinary open access archive for the deposit and dissemination of scientific research documents, whether they are published or not. The documents may come from teaching and research institutions in France or abroad, or from public or private research centers.
L'archive ouverte pluridisciplinaire $\mathbf{H A L}$, est destinée au dépôt et à la diffusion de documents scientifiques de niveau recherche, publiés ou non, émanant des établissements d'enseignement et de recherche français ou étrangers, des laboratoires publics ou privés. 


\title{
A Low-Cost System for Indoor Motion Tracking of Unmanned Aerial Vehicles
}

\author{
Sylvain Bertrand \#, Julien Marzat \#, Mathieu Carton \# \\ Cyril Chaix *, Paul Varela *, Renan Waroux *, Guillaume De Ferron*, Rosye Laurel* \\ \# ONERA - The French Aerospace Lab, F-91761 Palaiseau, France \\ $<$ firstname $>$.<lastname $>@$ onera.fr \\ ${ }^{*}$ Students, ECE, F-75015 Paris, France \\ $<$ lastname>@ece.fr
}

\begin{abstract}
This paper presents development and performance analysis of an indoor low-cost motion tracking system for miniature Unmanned Aerial Vehicles. The proposed system is based on infrared markers embedded on the vehicle, the positions of which are captured in real time by Wiimote infrared cameras. Using at least two of these cameras, vehicle 3D position and orientation can be determined. Implementation of system hardware architecture developed as student research project in academic environment is discussed with its own limitations. Estimation method is presented. System performance analysis is also proposed, addressing optimization of detection area, accuracy and dispersion of measurements, and robustness with respect to outliers and hardware parameters. Experimental results are provided to illustrate system performance and to demonstrate satisfaction of imposed strict constraints for reliable motion tracking of a miniature UAV.
\end{abstract}

\section{INTRODUCTION}

Miniature Unmanned Aerial Vehicles (UAVs) are widely used as dedicated robotic platforms to illustrate and to test performance of new algorithms and/or technologies. In particular, they can be used to validate navigation guidance and control algorithms more safely than on full scale aerial vehicles. Rotorcraft-based scale model vehicles are especially well suited for such easy-to-use test benches since they can be used in limited indoor areas thanks to their quasi-stationary flight capability.

The development of autonomous miniature UAV platform requires real time information on location and motion of the vehicle. These are commonly provided by embedded sensors such as accelerometers, rate gyros, magnetometers (combined into an Inertial Measurement Unit), GPS, baroaltimeter, range finders, etc. The measurements provided by these sensors are processed to estimate online vehicle state composed of its position, linear velocities, attitude angles and angular velocities.

To validate these estimation algorithms, further insight may be provided by external sensors delivering additional measurements that can be considered as references for comparison. Note that fusion techniques can also be used to directly take into account these measurements in state estimation process in order to improve its precision. For indoor experiments, motion capture systems are commonly used to provide additional measurements on position and on attitude of the vehicle. In this case, the vehicle is equipped by markers to be tracked. An acquisition system is then used to capture video frames from which a processing algorithm computes vehicle position and attitude by triangulation, knowing relative distances between markers.

Different technologies have been employed in motion capture systems. A first solution consists in using visible markers (such as colored objects or visible light sources) along with video cameras [1]-[6]. Image processing algorithms are hence applied to extract markers location in the video frames. Although efficient algorithms have been developed for image processing, such a solution may still be complex and particularly computer-demanding for real time trajectory tracking of a system with fast dynamics. To simplify markers localization process in video frames, solutions based on infrared (IR) may be preferable. Indeed, using an appropriate IR filter on a camera allows to capture only the markers in the videos frames without the remaining environment. Passive or active marker technologies can either be used for such a purpose. In the active solution, IR sources are directly disposed on the body to be tracked, while in the passive solution the body is equipped with reflective markers to be illuminated by infrared LEDs [7], [8]. A passive solution has been used in [9], [10] to determine the motion of a miniature UAV with very good accuracy and frequency. Nevertheless, usual cost of the system may also be rapidly prohibitive, especially when dealing with refined detection-data analysis as usually worked out. It is the intention in present paper to show that such a project can indeed be undertaken in more limited context of academic research without damaging results reliability. But then the price to pay is that further contradictory requirements have to be imposed onto the system. The motion tracking system must be low cost (a few hundred euros), easily movable from one indoor experimentation area to another one (i.e. small dimensions and mass, as well as easy calibration), and not vehicle-dependent. It must also provide position and attitude data with both frequency and precision sufficient enough to be used in a guidance loop process (at least $100 \mathrm{~Hz}$ ). Furthermore, the mass of marker system must be as light as possible, since additional payload mass is a critical issue for miniature UAVs. 

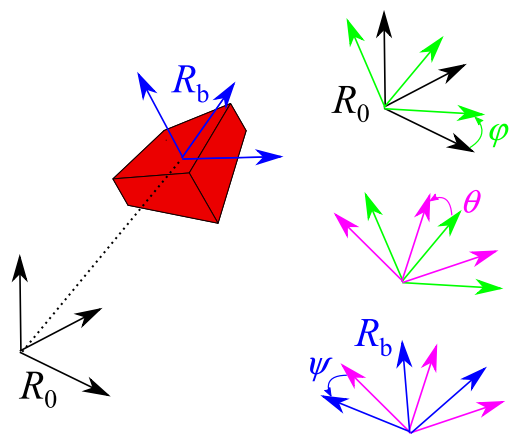

Figure 1: Transformation from inertial frame to body frame

To indicate that a solution does exist to this problem with a good compromise between additional constraints, the paper is organized as follows. In section II, the problem of motion tracking of such a rigid body by exogenous sensors is introduced, along with corresponding technical solution. In section III the hardware solution developed in the project is presented and robustness issues are then discussed in section IV. Experimental results are provided in section V to illustrate obtained performances. Concluding remarks and perspectives for future development are finally given in the last part of the paper. The major part of present work has been achieved by students of ECE Paris Graduate School of Engineering, in the context of a research project initiated by ONERA.

\section{EXOGENOUS MOTION TRACKING}

The aim of this work is to estimate from exogenous sensors the three-dimensional position and orientation of a rigid body, corresponding to the center of mass of a vehicle in motion, e.g., a robot or an UAV. The motion of such a mobile is classically parameterized in two different frames, namely a fixed inertial reference frame $R_{0}$ and a mobile body frame $R_{\mathrm{b}}$. The inertial frame is fixed and linked to the ground, while the body frame has its origin at the center of mass of the moving vehicle. The 3D position in inertial frame is denoted by $\mathbf{x}=[x, y, z]^{\mathrm{T}}$ and in body frame by $\mathbf{x}_{\mathrm{b}}=\left[x_{\mathrm{b}}, y_{\mathrm{b}}, z_{\mathrm{b}}\right]^{\mathrm{T}}$. Rotation of the body frame with respect to the inertial frame is generally parameterized by three Euler angles in roll, pitch and yaw, $\varphi, \theta, \psi$ (see Figure 1). Transformation from $\mathbf{x}_{\mathrm{b}}$ to $\mathbf{x}$ is given by the rotation matrix

$$
\mathbf{x}=\left[\begin{array}{ccc}
c_{\psi} c_{\theta} & -s_{\psi} c_{\varphi}+c_{\psi} s_{\theta} s_{\varphi} & s_{\psi} s_{\varphi}+c_{\psi} s_{\theta} c_{\varphi} \\
s_{\psi} c_{\theta} & c_{\psi} c_{\varphi}+s_{\psi} s_{\theta} s_{\varphi} & -c_{\psi} s_{\varphi}+s_{\psi} s_{\theta} c_{\varphi} \\
-s_{\theta} & c_{\theta} s_{\varphi} & c_{\theta} c_{\varphi}
\end{array}\right] \mathbf{x}_{\mathrm{b}}
$$

where $c_{\alpha}=\cos \alpha$ and $s_{\alpha}=\sin \alpha$.

The motion of the vehicle is then completely characterized by its pose, i.e., its position in inertial frame $[x, y, z]^{\mathrm{T}}$ and the three attitude angles $[\varphi, \theta, \psi]$, which should be estimated with high enough sampling rate, e.g., for control or observation purpose.

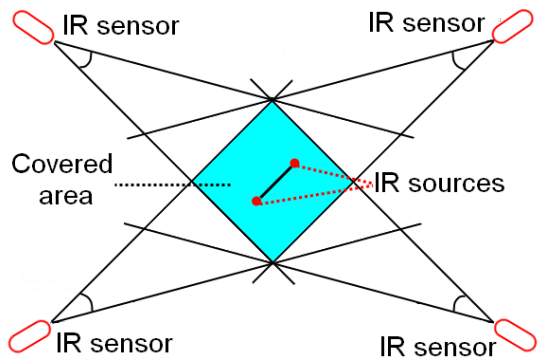

Figure 2: Tracking configuration

\section{A. Technical solution}

The proposed technical solution is to embed lightweight active IR sources on the vehicle and to use passive exogenous sensors to capture their motion. The minimal configuration required for complete six-degree-of-freedom tracking is composed of at least two sources and four sensors (see Figure 2). In this preliminary work, two sources and two sensors are taken into account, making it possible to estimate reliably position and attitude of a mobile in a half-space (see Figure 3 ). Extension to the general case of $N$ sources may be achieved by considering each couple of sensors separately and using adequate information fusion.

Infrared sources and cameras were preferred to standard visible image processing, since this technology makes it possible to achieve higher acquisition speed and easier identification of multiple sources.

\section{B. Estimation of position and attitude}

In this section, estimation of position and attitude is described considering that two IR cameras are available, tracking one or two IR sources (see Figure 3). The main tool used to reconstruct the three-dimensional position of each source is stereovision [11], [12].

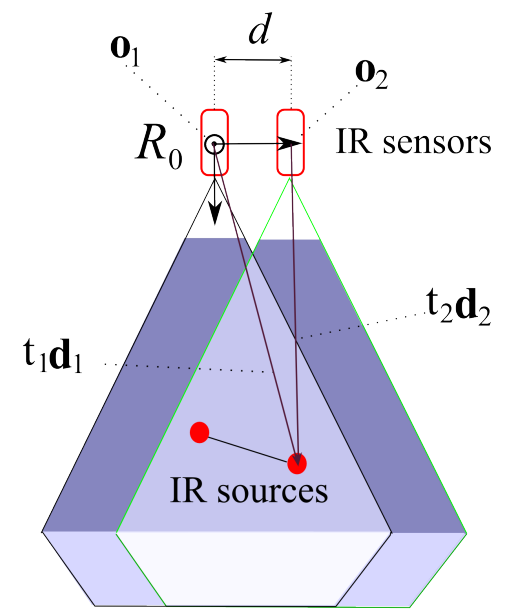

Figure 3: Tracking with 2 parallel IR sensors

The inertial reference frame is linked to one of the sensors, its origin being denoted by $\mathbf{o}_{1}$. The location of the second sensor in this frame is denoted by $\mathbf{O}_{2}$, while the coordinates 


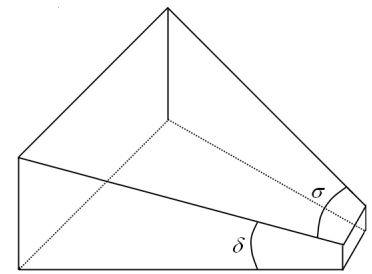

Figure 4: IR sensor field of view

of the point to be tracked is $\mathbf{x}=[x, y, z]^{\mathrm{T}}$ in $R_{0}$. In the parallel configuration of Figure $3, \mathbf{o}_{1}=[0,0,0]^{\mathrm{T}}$ and $\mathbf{o}_{2}=[d, 0,0]^{\mathrm{T}}$.

1) Position: The two rays $\mathbf{r}_{1}$ and $\mathbf{r}_{2}$ are two different ways to represent the unknown $3 \mathrm{D}$ position of the point of interest. They can be decomposed into

$$
\left\{\begin{array}{l}
\mathbf{r}_{1}=\mathbf{o}_{1}+t_{1} \mathbf{d}_{1} \\
\mathbf{r}_{2}=\mathbf{o}_{2}+t_{2} \mathbf{d}_{2}
\end{array}\right.
$$

where $\mathbf{d}_{1}$ and $\mathbf{d}_{2}$ are direction vectors from each sensor to the source, while $t_{1}$ and $t_{2}$ are the unknown distance between the source and each sensor in these directions. Each camera measures two coordinates in its two-dimensional focal plane, denoted respectively by $\left[x_{1}, y_{1}\right]$ for the first one and $\left[x_{2}, y_{2}\right]$ for the second one. The resolution of focal plane being $1024 \times$ 768 , mapping of these coordinates to $[-1 ; 1]$ is

$$
\left\{\begin{aligned}
\widehat{x}_{i} & =\frac{2 \cdot x_{i}}{1024}-1 \\
\widehat{y}_{i} & =\frac{2 \cdot y_{i}}{768}-1
\end{aligned}\right.
$$

for $i=1,2$. This now allows to compute the direction vectors $\mathbf{d}_{1}$ and $\mathbf{d}_{2}$. The field of view of the Wiimote, as displayed in Figure 4, is assumed to be parameterized by two angles $\sigma$ and $\delta$, horizontally and vertically. Since the sensor is assumed to point toward $[0 ;-1 ; 0]^{\mathrm{T}}$, the coordinates of each ray direction are given by

$$
\mathbf{d}_{1}=\left[\begin{array}{c}
\widehat{x}_{1} \cdot \tan \left(\frac{\sigma}{2}\right) \\
1 \\
\widehat{y}_{1} \cdot \tan \left(\frac{\delta}{2}\right)
\end{array}\right], \mathbf{d}_{2}=\left[\begin{array}{c}
\widehat{x}_{2} \cdot \tan \left(\frac{\sigma}{2}\right) \\
1 \\
\widehat{y}_{2} \cdot \tan \left(\frac{\delta}{2}\right)
\end{array}\right]
$$

There remains two unknown constants $t_{1}$ and $t_{2}$, which can be determined by equating $\mathbf{r}_{1}$ and $\mathbf{r}_{2}$ as

$$
\mathbf{o}_{1}+t_{1} \mathbf{d}_{1}=\mathbf{o}_{2}+t_{2} \mathbf{d}_{2} \text {. }
$$

Computing the cross product (since the problem is in a $3 \mathrm{D}$ space) of this expression by $\mathbf{d}_{1}$ yields

$$
\mathbf{o}_{1} \times \mathbf{d}_{1}=\mathbf{o}_{2} \times \mathbf{d}_{1}+t_{2} \mathbf{d}_{2} \times \mathbf{d}_{1}
$$

and finally

$$
t_{2}=\frac{\left\|\mathbf{o}_{1} \times \mathbf{d}_{1}-\mathbf{o}_{2} \times \mathbf{d}_{1}\right\|}{\left\|\mathbf{d}_{2} \times \mathbf{d}_{1}\right\|}=\frac{\left\|\left(\mathbf{o}_{1}-\mathbf{o}_{2}\right) \times \mathbf{d}_{1}\right\|}{\left\|\mathbf{d}_{2} \times \mathbf{d}_{1}\right\|} .
$$

Similarly, computing the cross product of (5) by $\mathbf{d}_{2}$ gives

$$
t_{1}=\frac{\left\|\left(\mathbf{o}_{2}-\mathbf{o}_{1}\right) \times \mathbf{d}_{2}\right\|}{\left\|\mathbf{d}_{1} \times \mathbf{d}_{2}\right\|} .
$$

The three coordinates of $\mathbf{r}_{1}$ and $\mathbf{r}_{2}$ can thus be determined with (4), (7) and (8), which gives two different estimates of $\mathbf{x}$ that can be used separately or in combination as $\widehat{\mathbf{x}}=$ $\left(\mathbf{r}_{1}+\mathbf{r}_{2}\right) / 2$.

2) Attitude: Position tracking of two different IR sources $\mathbf{x}^{(1)}$ and $\mathbf{x}^{(2)}$, as described above, is required for attitude estimation. A simple way to obtain $\varphi, \theta, \psi$ is to consider that the initial orientation of the vector $\mathbf{x}^{(1)}-\mathbf{x}^{(2)}$ with respect to inertial reference frame is known (generally zero). Denoting this initial position vector by $\mathbf{m}_{0}$, an estimate of attitude angles can be obtained by computing the cross-product at each time step of the new position vector $\mathbf{x}^{(1)}-\mathbf{x}^{(2)}$ with $\mathbf{m}_{0}$ and taking its arcsine component per component. Note that the distance between the two points $\mathbf{x}^{(1)}$ and $\mathbf{x}^{(2)}$ can remain unknown without prejudice.

\section{HARDWARE SOLUTION}

\section{A. Architecture}

The system is composed of three different parts: the marker system, the IR acquisition system and the data processing system. Let us recall that the main constraints are that the motion tracking system must be low cost, easy to be moved and calibrated, and easy to be adapted on different vehicles. These constraints have strongly oriented the choice of hardware solutions for each of the three different parts of the system.

1) The marker system: The marker system consists of IR sources that will be fixed to the vehicle. Each source is composed of seven IR LEDs mounted on a PCB and oriented with different inclinations to increase the domain of visibility of the source. As can be seen on Figure 5, different types of IR LEDs have been selected depending on their characteristics. Three LEDs with a narrow beam of 44deg and intensity of $350 \mathrm{~mW} / \mathrm{sr}$ have been disposed at the center of the source, whereas four LEDs with larger beams of 60deg but with intensity of $200 \mathrm{~mW} / \mathrm{sr}$ are disposed at the edges of the PCB. This choice has been made for a good trade-off between power and angles in order to create a source being visible from many different directions.

Note that although seven LEDs are used, they will be seen as a unique IR source by the acquisition system (see section III-A2).

2) The IR acquisition system: The choice of IR acquisition system has been strongly impacted by the low cost constraint. A first solution may have consisted in using a webcam along

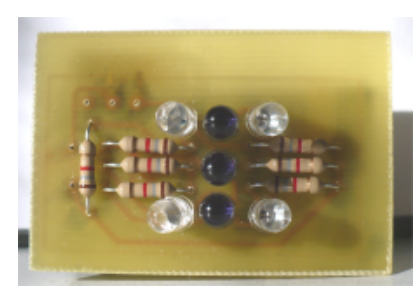

Figure 5: Each marker is composed of seven IR LEDs 
with an infrared filter. But in this case, extraction of detected sources from the image may still require some effort.

Nintendo Wii's remote controllers, Wiimotes, are equipped with an infrared camera that can detect up to 4 infrared sources and directly transmit their coordinates in an image frame of $1024 \times 768$ pixels with a frequency up to $1 \mathrm{kHz}$. Some research projects have already demonstrated the feasibility of the use of a Wiimote as an IR acquisition system [13], even for fast dynamical systems [14]. The low cost of a Wiimote controller makes it very well suited for its use in present project.

Acquisition of the IR sources coordinates delivered by the Wiimotes is achieved by a PC equipped with a Bluetooth connection and using the open source library Wiiuse [15].

3) The data processing system: Acquisition of IR sources coordinates and the data processing are performed on the same Linux PC, by a software developed in $\mathrm{C}$ language.

This software handles the different following tasks:

- calibration of different system parameters by the user (number of Wiimotes and of IR sources to be tracked, distance between the Wiimotes, aperture angles of Wiimote IR cameras, etc.),

- connection of the Wiimotes and identification of the IR sources to be tracked,

- acquisition and data processing : acquisition of IR sources coordinates in the camera frame and filtering (see section IV-D), computation of the 3D coordinates of each IR source, save in a data file.

The main task (acquisition and data processing) runs at a frequency of $100 \mathrm{~Hz}$. This frequency could be increased (up to $1 \mathrm{kHz}$ frequency allowed by the Wiimotes) if required. A median temporal filter has been used to increase robustness to outliers.

\section{PERformanCE ANALYSis}

\section{A. Sensor location to optimize coverage}

Stereovision requires that both sensors (in a two-IR-camera configuration) view the sources to be located at each time step. To maximize the area covered by the system, the different sensors should be optimally positioned [13], [16]. In this section, distance and rotation angles between sensors are investigated so as to maximize this area.

With two parallel IR cameras, and since the maximum range of the sensors is 5 meters, the horizontally area covered and the minimum distance of acquisition (see Figure 6) are

$$
\begin{gathered}
\mathcal{A}_{\text {parallel }}=\left(5 \cos \left(\frac{\sigma}{2}\right)-\frac{d}{2 \cdot \tan \left(\frac{\sigma}{2}\right)}\right)^{2} \cdot \tan \left(\frac{\sigma}{2}\right) \\
p=\frac{d}{2 \cdot \tan \left(\frac{\sigma}{2}\right)}
\end{gathered}
$$

where the parameters $\sigma, d$ and $p$ are introduced on Figure 6 .

The maximum area is thus obtained for the smallest distance possible between sensors (limited by the dimensions of cameras) and is equal to $3.4 \mathrm{~m}^{2}$ with $\sigma=33^{\circ}$, which also reduces $p$ to a very small value. No area is covered for $d>10 \sin (\sigma / 2)$.

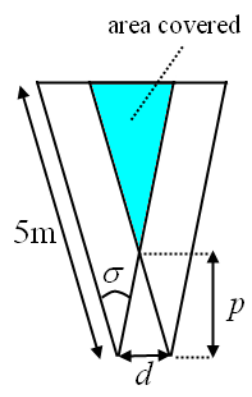

(a) Geometry

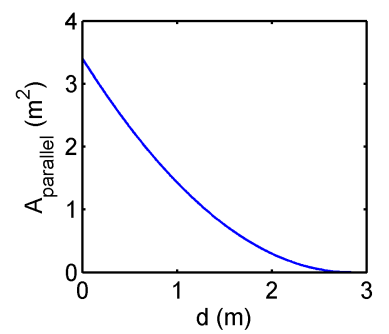

(b) Visible area depending on $d$
Figure 6: Parallel configuration

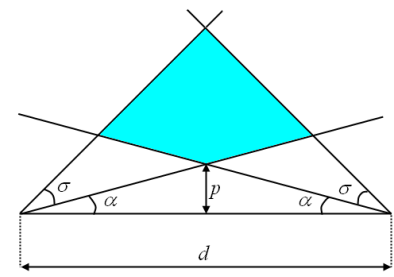

(a) Geometry

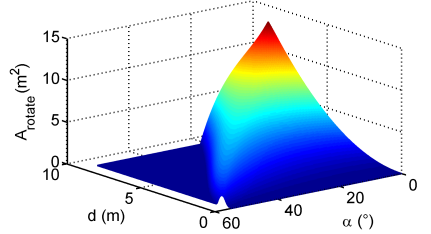

(b) Visible area depending on $\alpha$ and $d$
Figure 7: Rotated configuration

To increase coverage, the sensors may be rotated by an angle $\alpha$, as shown in Figure 7. The area covered is then

$$
\mathcal{A}_{\text {rotate }}=\frac{d^{2}(\tan (\alpha+\sigma)-\tan (\alpha))^{2}}{4(\tan (\alpha+\sigma)+\tan (\alpha))}
$$

The maximal allowed angle $\alpha$ for each distance $d$ is $\alpha_{\max }=\arccos (d / 10)-\sigma$, with a maximum range of 5 meters. According to the $3 \mathrm{D}$ plot of visible area $\mathcal{A}_{\text {rotate }}$ as a function of $d$ and $\alpha$, the maximum visible area is obtained for $\alpha=0$ and for a maximal distance between the sensors, which is also limited by the constraint of 5-meter range. For $\sigma=33^{\circ}$, this maximum distance is equal to $d=8.4 \mathrm{~m}$ and provides a visible area of $11.4 \mathrm{~m}^{2}$. This also yields $p=0 \mathrm{~m}$. Note that the maximum visible area is always located at $\alpha_{\max }$, for any fixed $d$.

\section{B. Robustness with respect to field of view angles}

Determination of the 3D coordinates of IR sources is strongly affected by errors on the values of the angles $\sigma$ and $\delta$ defining horizontal and vertical fields of view of the IR camera. Different values for these two angles can be found in the literature [13], [17]. In addition they may vary from one Wiimote to an another one. Therefore it is necessary to estimate the value of these angles corresponding to each hardware device.

Experiments have been conducted to estimate these angles for the two Wiimotes used for our platform, by simply finding the limits of the field of view with an IR sources, located at different distances from the camera. From these experiments the mean values $\sigma=31^{\circ}$ and $\delta=38.5^{\circ}$ have been found. 


\section{Accuracy and dispersion}

To quantify the accuracy and the noise of the measured values, the following experiment has been conducted. Acquisition of the planar motion of two sources separated by a fixed distance moving in the range of the IR cameras has been done with 8000 points. The Euclidian norm between their positions has then been computed, and Figure 8 shows measurements histogram. Assuming that $\mathbf{x}$ and $\mathbf{y}$ axis suffer Gaussian noise of standard deviation $\sigma_{\mathrm{R}}$, then their $\mathcal{L}_{2}$ norm follows a Rayleigh distribution of parameter $\sigma_{R}$. The maximum-likelihood estimation of this parameter on available data gives $\sigma_{\mathrm{R}}=0.85$, which means that the standard deviation of the original noise is approximately $0.8 \mathrm{~cm}$.

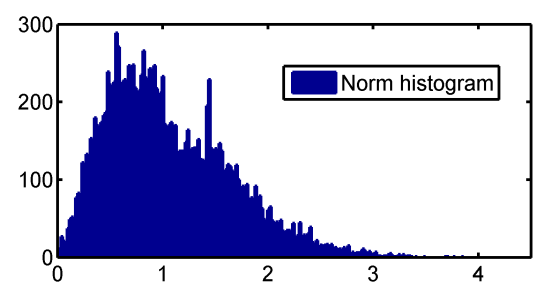

Figure 8: Measurement histogram

\section{Robustness with respect to outliers}

Data acquired by Wiimotes are subject to significant outliers which may result in very perturbed positions of IR sources, if not filtered. A simple filter has been implemented by a two step approach. At the beginning of acquisition, positions of IR sources are stored for a few seconds. A mean position is hence computed to get stable locations of each IR source to be tracked. Then, only the data in a neighborhood of the last position are considered as input for data processing. The size of this neighborhood is defined in terms of vertical and horizontal pixels of a squared window in the camera frame, centered on the last position of the IR source. This size can be adjusted by the user during calibration step.

Very good results have been obtained by this simple filtering procedure, which also eliminates parasitic measurements possibly due to apparition of perturbing new IR sources that are not to be tracked.

\section{EXPERIMENTAL RESULTS}

\section{A. Setup}

To illustrate the performance obtained with the system developed in this study, an experiment was conducted to track an object moving in a plane. This corresponds to the application of a miniature UAV flying at constant altitude. Two IR sources were fixed on a rigid body, at the same distance from its center of gravity. The objective consisted in tracking the two dimensional position of the center of gravity in the considered plane as well as the angle defining the body orientation in this plane.

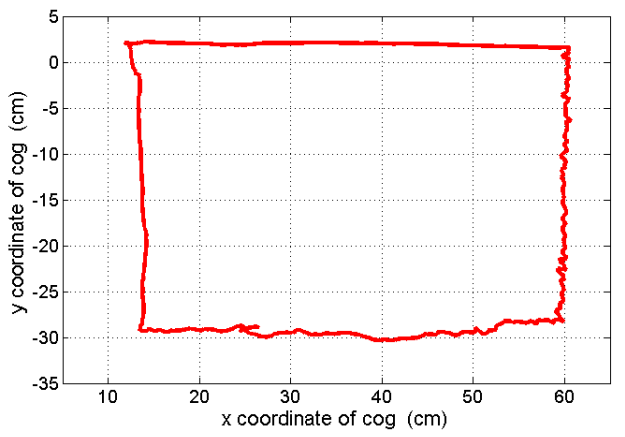

Figure 9: Position of the center of gravity $(\operatorname{cog})$ of the body tracked while following a rectangular trajectory

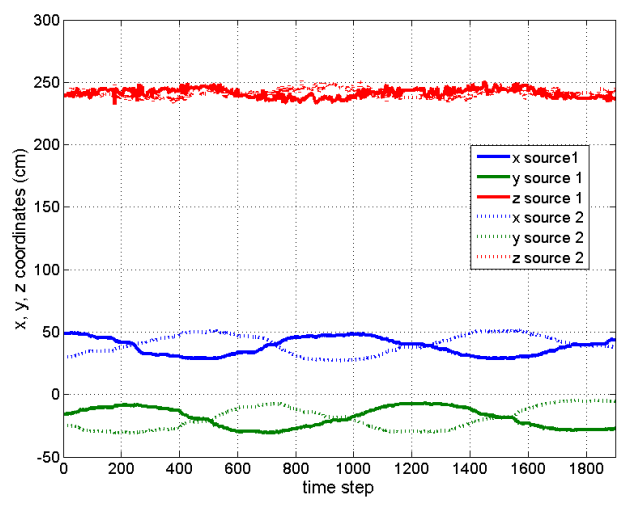

Figure 10: Coordinates of the IR sources during rotation of the body to be tracked

\section{B. Results}

In a first experiment, the object to be tracked was moved on a linear trajectory along a rectangle of $30 \mathrm{~cm}$ by $50 \mathrm{~cm}$. The body orientation has not been changed during the experiment. Figure 9 presents evolution of the coordinates of center of gravity in the plane of motion, computed from coordinates estimated for the two IR sources. As it can be seen, the dimensions of the rectangle described by estimated center of gravity of the body, here about $32 \mathrm{~cm}$ by $46 \mathrm{~cm}$, roughly match the dimensions of initial fixed rectangle. Differences can be explained by accuracy issues considered in sections IV-B to IV-D, by distortion issues in the focal plane of the sensors that have not been addressed in this study, as well as by positioning errors of the body along the reference trajectory during the experiment. Owing to the very stringent low cost constraint on the system, this experiment shows however good performance in linear tracking of the rigid body.

A second experiment was conducted to illustrate system performance for angular motions. The body was rotated around its center of gravity along an axis perpendicular to the plane of motion. Coordinates of the two IR sources have been computed, and their evolutions are plotted with respect to time step on Figure 10 (each time step corresponds to a period of time of $0.01 \mathrm{~s})$. The $z$-coordinate of each source can be considered as constant with respect to time, illustrating the fact that the motion is nearly planar. 


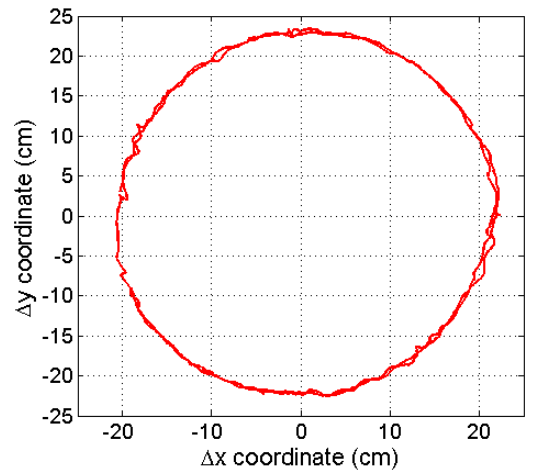

Figure 11: Coordinates of the difference between the position vectors of the two IR sources during rotation of the body to be tracked

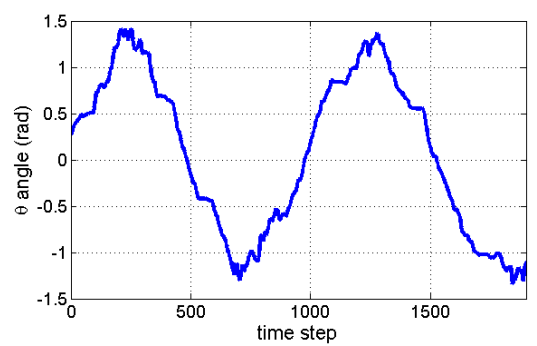

Figure 12: Evolution of the estimated angle of the rigid body during rotation

It can be verified that the difference between position vectors of the two sources follows a circular trajectory in a plane, as presented in Figure 11. The body rotation angle $\theta$ has also been computed from IR sources positions and its evolution with respect to time is presented on Figure 12. Positionning errors of IR sources lead to errors in the angle computation, as it can be seen that $\theta$ never reaches the value of $\pm \pi / 2$ although complete rotations of the body have been performed around its center of gravity. This can also be explained by the fact that body motion was experimentally not exactly planar. Nevertheless, tracking performance in the case of angular motions can also be considered as quite satisfactory.

\section{CONCLUSIONS AND PERSPECTIVES}

Design and performance analysis of a low cost motion tracking system have been discussed in this paper. Based on Wiimote cameras and on infrared markers disposed on the body to be tracked, it has been shown how body position and attitude can be reliably estimated. The system developed in this study completely enables motion tracking at $100 \mathrm{~Hz}$ frequency. Hardware solution has been presented and different issues have been analyzed regarding coverage optimization, robustness with respect to hardware parameters and measurement outliers. System accuracy has also been analyzed. Several experimental results have then been presented to illustrate the very good performance of the proposed system.

The motion tracking system developed in this project also fulfills initially imposed low cost constraint, and makes it remarkably well suited for further study in academic environment. Among future improvements, an auto calibration procedure will be studied, based on callibration pattern, as for example presented in [18].

\section{ACKNOWLEDGEMENTS}

The authors are very much indebted to Prof. M. Cotsaftis (LACSC/ECE) for advice and coordination of this publication.

\section{REFERENCES}

[1] K. Kondak, M. Bernard, N. Meyer, and G. Hommel, "Autonomously Flying VTOL-Robots: Modeling and Control," in Proceedings of the 2007 IEEE International Conference on Robotics and Automation, Roma, Italy, 2007, pp. 736-741.

[2] D. Won, H. Oh, S. S. Huh, D. H. Shim, and M. J. Tahk, "Multiple UAVs Tracking Algorithm with a Multi-Camera System," in Proceedings of International Conference on Control, Automation and Systems 2010, Gyeonggi-do, Korea, 2010, pp. 2357-2360.

[3] H. Oh, D. Y. Won, S. S. Huh, D. H. Shim, and M. J. Tahk, "Experimental Framework for Controller Design of a Rotorcraft Unmanned Aerial Vehicle Using Multi-Camera System," International Journal of Aeronautical \& Space Sciences, vol. 11, pp. 69-79, 2010.

[4] M. Achtelik, T. Zhang, K. Kühnlenz, and M. Buss, "Visual tracking and control of a quadcopter using a stereo camera system and inertial sensors," in Proceedings of IEEE International Conference on Mechatronics and Automation, Changchun, China, 2009.

[5] E. Altug, J. P. Ostrowski, and R. Mahony, "Control of a Quadrotor Helicopter Using Visual Feedback," in Proceedings of the 2002 IEEE International Conference on Robotics \& Automation, Washington, USA, 2002, pp. 72-77.

[6] P. Xu, UAV Position and Attitude Sensoring in Indoor Environment using Cameras. Department of Electrical and Computer Engineering, Clemson University.

[7] M. Ribo, A. Pinz, and A. Fuhrmann, "A new optical tracking system for virtual and augmented reality applications," in Proceedings of the 18th IEEE Instrumentation and Measurement Technology Conference, Budapest, Hungary, vol. 3, 2001, pp. 1932-1936.

[8] S. Attygalle, M. Duff, T. Rikakis, and J. He, "Low-cost, at-home assessment system with Wii Remote based motion capture," in Proceedings of Virtual Rehabilitation, 2008, 2008, pp. 168-174.

[9] N. Kundak and B. Mettler, "Experimental Framework for Evaluating Autonomous Guidance and Control Algorithms for Agile Aerial Vehicles," in Proceedings of the European Control Conference 2007, Kos, Greece, pp. 293-300.

[10] M. Valenti, B. Bethke, G. Fiore, and E. How, J. P.and Feron, "Indoor Multi-Vehicle FlightTestbed for Fault Detection, Isolation, and Recovery," in Proceedings of the 2006 AIAA Guidance, Navigation, and Control Conference and Exhibit, Keystone, USA, 2006.

[11] M. P. Wronski, Design and Implementation of a Hand Tracking Interface using the Nintendo Wii Remote. Department of Electrical Engineering, University of Cape Town, 2008.

[12] S. De Amici, A. Sanna, F. Lamberti, and B. Pralio, "A Wii remote-based infrared-optical tracking system," Entertainment Computing, 2010.

[13] S. Hay, J. Newman, and R. Harle, "Optical tracking using commodity hardware," in Proceedings of the 7th IEEE/ACM International Symposium on Mixed and Augmented Reality (ISMAR), Cambridge, UK, 2008, pp. $159-160$

[14] K. E. Wenzel, A. Masselli, and A. Zell, "Automatic Take Off, Tracking and Landing of a Miniature UAV on a Moving Carrier Vehicle," Journal of intelligent \& robotic systems, pp. 1-18, 2011.

[15] "http://www.wiiuse.net/docs/."

[16] T. Cuypers, T. Van den Eede, S. Ligot, Y. Francken, C. Hermans, F. Arickx, and P. Bekaert, "StereoWiision: Stereo Vision with Wiimotes,' International 3D Stereo Film and Technology Festival (3D Stereo MEDIA), Liège, Belgium, 2009.

[17] J. C. Lee, "Hacking the nintendo wii remote," IEEE Pervasive Computing, pp. 39-45, 2008.

[18] D. Wang and D. Huang, Low-Cost Motion Capturing Using Nintendo Wii Remote Controllers. Project Report, Department of Electrical and Computer Engineering, University of Toronto, 2008. 